\section{COAGULATION OF COLLOIDS}

\author{
By Prof. ERIC K. RIDEAL, M.B.E., F.R.S.
}

$\mathrm{I}^{\mathrm{N}}$ $\mathrm{T}$ his presidential address delivered to the Chemistry Section of the Indian Science Congress last January, Prof. S. S. Joshi, of the Benares Hindu University, discussed inter alia various aspects of the kinetics of colloid systems.

In dealing with molecular reactions in solution, three methods of attack have yielded interesting and important results; one based upon gas kinetic concepts, the second on the Einstein diffusion equations and, finally, the transition state method of approach. In reactions between colloid particles or macromolezules, the second method of approach is generally employed and we are indebted to Smoluchowski for formulating the general principles.

The rates of colgulation are arbitrarily divided into rapid and slow rates respectively, the latter being derived from the former by the introduction of a fuctor $\xi$ giving the fruction of collisions which are 'successful'. Since the rate of coagulation falls as particle charge rises, the potential barrier due to the diffuse layer of ions around the particles (the zeta potential $\zeta$ ) can be regarded in the light of a potential barrier to be overcome for succes sful collision; we would thus anticipate that an equation of the type

$$
\log \xi=\frac{K \zeta}{R T}+C
$$

should connect the collision factor $\xi$ with the zeta potential $\zeta$. This is true for certain cases. We have found it to hold for quartz covered with gliadin, but in many cases the rate of coagulation is independent of $\zeta$ over a considerable range. Wiegner, Tuorila and Müller have shown that Smoluchowski's equations can be applied with considerable success to the problem of coagulation of uncharged particles, and extended the work to anisodimensional particles and more particularly to polydisperse systems. In the latter case it was shown that if the system were in motion the larger particles would 'strain out' the smaller ones. We might anticipate that the walls of the containing vessel would serve as collectors to neutral colloid particles undergoing auto-coagulation if the suspension was kept agitated. Prof. Joshi cites many cases of the effect of the containing vessel on the coagulation rates of sols, and makes the important olsservation that it is not necessary that the wall should have a zeta potential opposite in sign to that of the colloid.

Prof. Joshi rightly directs attention to the fact that the kinetics of coagulation do not obey the simple theory. The work of Levine in Great Britain and of the Amsterdam school of investigators has shown that for charged particles a position of minimum potential energy can be found in which the p.rticles are a finite distance apart. We might thus expect to find doublets present in solution, which would require a definite energy of activation before they underwent true coagulation or coalescence; here the coxgulation-rate would be pseudo-unimolecular. Another dificulty, which was first emphasized by Haber, is that the particles on coalescence undergo the phenomenon of 'ageing', resulting in a change of size and consequently a change in the diffusion coe ficient on which the kinetics of coagulation are dependent. This ageing process, which can be followed in miny cases by means of $\mathrm{X}$-rays or by the rate of liberation of absorbed emanation or dyes, is virtually a process of recrystallization and proceeds most rapidly in heteropolar systems such as colloidal sodium chloride, and much more slowly in colloids such as the hydrous oxides or sulphides.

The most satisfactory method of determining the rate of coagulation of a colloidal solution is by direct observation with the aid of an ultra-microscope. This method is eminently suitable for colloidal systems in which the refractive index of the disperse phase is markedly different from that of the dispersion medium; but where the light scattering is poor, as is the case for the proteins or hydrous oxides, many observational errors are apt to creep in. Many observers have consequently employed more indirect methods, espesially the viscosity. The Benares school has made much use of refractivity measurements for this purpose.

The utilization of viscosity measurements for following the rate of coagulation may be seriously called in question. To a first approximation the vis. cosity of a sol. if the electroviscous effect be eliminated by the addition of an electrolyte, should, in accord. ance with Einstein's equation, be independent of the dispersity of the disperse phase, and only depend on its total volume. Prof. Joshi states that the viscosities of a number of dilute emulsions likewise obey the Einstein equation. This can scarcely be the case if the disperse phase has a measurable viscosity per se. Prof. G. I. Taylor, some years ago, evaluated the viscosity of a dilute emulsion in terms of the volume fractions and specific viscosities of the two phases, but so far as I am aware, this equation has not been subjected to any detailed experimental verification. Changes in viscosity do, however, occur in coagulation of colloidal systems, partly because the specific volume of the disperse phase changes due to dehydra. tion, partly because a shape factor begins to emerge as the particles grow and, in some cases at least, as investigations on solutions of polymers have re. vealed, the larger particles may be deformable under shear and thus raise the viscosity.

It is evident that many factors enter into the problem of the kinetics of coagulation and their determination, and we are indebted to Prof. Joshi and his school at Benares for much painstaking and detailed work in this difficult experimental field. It is satisfactory to note that real progress is being made in that the factors operative are being isolated and identified.

\section{ANGLO-PORTUGUESE SCIENTIFIC RELATIONS \\ By SIR . GEORGE THOMSON, F.R.S.}

T $N$ view of the renewed intimacy which recent 1 events have infused into the centuries-old associs. tion of Portugal and Great Britain, and of the pros. pect of increased co-operation between the two countries, there is a special significance in the publication of a study in Portuguese by Prof. H Amorim Ferreira on Anglo-Portuguese scientifio relations*. The history of science is often treated as if it were a matter of a few great names, and one is apt not to realize the importance of the part played by those whose fame was greater in their life-time than in after ages. In particular, problems of the

- Relaçõ is cientícas entre Portugal e a Gi a.Bretanha. Por H. Amorim Ferreira. Pp. viii +76 . (Lisbon: Academia das Ciencias de Lisboa, 1943.) 